\title{
Diversity Within the Most Competitive Internal Medicine Fellowships: Examining Trends from 2008 to 2018
}

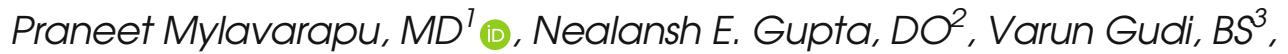 \\ Apoorva Mylavarapu, BS ${ }^{4}$, Lori B. Daniels, MD, MAS ${ }^{7}$, and Mitul Patel, $\mathrm{MD}^{7}$
}

'Department of Cardiology, University of California, San Diego, CA, USA; ${ }^{2}$ SUNY Downstate Medical Center, New York, NY, USA; ${ }^{3}$ St. George's University, St. George's, Grenada; ${ }^{4}$ David Geffen School of Medicine, University of California, Los Angeles, CA, USA.

BACKGROUND: Prior studies have demonstrated the importance of diversity among physicians. Identifying trends in diversity within the most competitive internal medicine (IM) fellowships can guide focused efforts to address barriers to equal representation.

OBJECTIVE: To examine the racial and gender composition of resident applicants and accepted fellows to the top five most competitive IM specialties.

DESIGN: Survey data from the AAMC, JAMA, and NRMP were obtained. Fisher's exact tests were conducted to compare differences in representation between fellows in the most competitive specialties, resident applicants into those specialties, and categorical IM residents. Linear regression was used to analyze trends within each group.

PARTICIPANTS: Categorical IM residents and fellows at ACGME-accredited M.D. programs in the USA.

MAIN MEASURES: Proportion of each population by gender and race/ethnicity

KEY RESULTS: Women saw an increase in representation among accepted fellows to the most competitive IM fellowships from 2008 to $2013(+4.4 \%, p<0.011)$, but the trend has since plateaued at a level (34\%) significantly lower than their representation among IM residents $(43 \%, p<0.001)$. Black representation among accepted fellows (4.6\%) has been increasing from 2008 to $2018(+1.2 \%, p=0.001)$, but is still significantly lower than their representation among IM residents $(5.6 \%, p<0.001)$. Hispanic resident applicant and fellow representation have seen minimal change.

CONCLUSION: Despite trends towards better representation among women and underrepresented minorities (URMs) among fellows in the most competitive IM specialties from 2008 to 2013, there has been a stagnation in both gender and racial diversity over the past 5 years. Further efforts must be undertaken to address barriers to entry and advocate for better representation of women and URMs in fellowship programs.

KEY WORDS: diversity; medical education; fellowship; residents; medical students.

J Gen Intern Med 35(9):2537-44

DOI: $10.1007 / \mathrm{s} 11606-020-06008-5$

(c) Society of General Internal Medicine 2020

\section{Prior Presentation: None}

Received October 6, 2019

Revised March 24, 2020

Accepted June 23, 2020

Published online July 14, 2020

\section{BACKGROUND}

In the face of an increasingly diverse population in the USA, the physician workforce has failed to adapt. While racial and gender diversity among medical school graduates has increased since 1975, this trend has plateaued from 2012 onwards with underrepresented racial minorities (URMs) representing less than one-quarter of the annual pool of graduates. ${ }^{1}$ This is further compounded by a stark decrease in the percentage of full-time faculty positions and department chairs within academic medicine held by racial and gender minorities. ${ }^{2}$

Numerous studies have demonstrated racial inequalities in health care in the USA. And while the causes of these disparities are complex, there is significant evidence that racial bias in the healthcare system is a major factor. For example, Black patients with Black physicians reported higher levels of trust in their physicians and increased utilization of routine checkups, mammograms, and other preventive care services. ${ }^{3}$ Similarly, Black and Hispanic patients with racially concordant providers reported high rates of excellent care, preventive care utilization, and greater overall satisfaction. ${ }^{4}$

While these findings are not meant to argue for medical segregation or minimize the efforts of non-minority physicians who support URMs, it is increasingly clear that there is a large unfilled need for minority physicians in the workforce. ${ }^{5,6}$

Despite support for workforce diversification from the AAMC and Institute of Medicine, there has been little improvement in representation across the pipeline, ${ }^{7}$ indicating a need to examine and address barriers within the training process.

Internal medicine (IM) continues to establish itself as one of the most popular fields for graduating medical students. In 2019 alone, over $20 \%$ of all residency applicants applied to categorical postgraduate year 1 (PGY1) positions offered in IM. ${ }^{8}$ Furthermore, over two-thirds of PGY3 categorical residents annually apply to fellowships as a result of increasing interest in specialized medicine. ${ }^{9}$ Efforts have been made by leaders in IM to address the gap in representation at multiple levels of recruitment. ${ }^{10,11}$ However, racial and gender disparity remains apparent among physicians in fields such as gastroenterology, cardiology, and critical care medicine. ${ }^{12-14}$

While there have been many studies observing gender and racial disparities within medicine, to date no study has 
compared these trends among resident applicants to fellowship and accepted fellows within IM specialties. Thus, the purpose of our study was to examine the racial and gender composition of applicants and accepted fellows to the top five most competitive IM specialties.

\section{METHODS}

Data regarding the 2018 fellowship match for IM specialties were obtained from the National Residency Matching Program (NRMP). The five most competitive specialties, defined as having the greatest number of applicants for each filled fellow position, were selected for further analysis. In order, the five most competitive fellowships were Gastroenterology (GI), Rheumatology (RHEUM), Cardiovascular Diseases (CVD), Pulmonary Disease and Critical Care Medicine (PCCM), and Hematology and Oncology (HEME). Aggregated specialty data were obtained from the Association of American Medical Colleges' (AAMC) Electronic Residency Application Services (ERAS) from 2007 to 2018 for each of the five specialties. ${ }^{15}$ Data obtained included a breakdown of applicants to each Accreditation Council for Graduate Medical Education (ACGME) fellowship program by gender and race/ethnicity. Corresponding data for residents applying into these specialties were obtained from the Journal of the American Medical Association (JAMA) Graduate Medical Education annual reports. ${ }^{16-25}$ Corresponding data for accepted IM residents were also obtained from JAMA. Variables included gender and race/ethnicity.

Groups analyzed included self-identified Black, Hispanic, and female residents, and fellows as these groups have historically been racial, ethnic, and gender minorities in medicine.

Analysis was performed separately for each specialty, and also at an aggregate level for all five specialties combined. Binomial distribution analysis was used to assess the minority subgroup representation among resident applicants compared with the corresponding representation among fellows. Twotailed Fisher's exact tests were performed between resident applicants and corresponding fellows for each minority subgroup within each specialty for every year from 2008 to 2018 . Comparisons were set up with the null hypothesis indicating the fellow representation was significantly different from the resident applicant representation. The proportions of resident applicants and fellows in each specialty were plotted by race and gender from 2008 to 2018. Linear regressions were performed for resident applicants and fellows, and best fit trendlines with 95\% confidence bands were plotted for each specialty.

Statistical analysis was conducted using RStudio (RStudio, Boston, MA). This study was IRB exempt.

\section{RESULTS}

The data were analyzed by combining the top five most competitive specialties (GI, RHEUM, CVD, PCCM, HEME) to examine overall trends across these selective specialties.

\section{Gender Analysis}

Female resident applicants and fellows in the most competitive IM specialties made up $37.5 \%$ and $36.2 \%$ of each group respectively in 2018 (Table 1; Fig. 1). The overall female proportion of fellows significantly increased from 2008 to 2018 at a rate of $0.3 \%$ per year $(p$ trend $=0.02$ ). However, most of this increase occurred between 2008 and 2013, at a rate of $0.9 \%$ per year ( $p$ trend $=0.007$ ). From 2014 to 2018, female representation plateaued, with no significant change in the overall female proportion of fellows during this time ( $p$ trend $=0.15$ ). Furthermore, from 2008 to 2018 , there was no significant change in the overall female proportion of resident applicants ( $p$ trend $=0.98$ ) (Fig. 2). Female representation decreased when moving from IM residency to fellowship in the most competitive fields $(p<0.001)$ across the same time period (Fig. 3).

\section{Race and Ethnicity Analysis}

The overall proportion of Black fellows significantly increased from 2008 to 2018 at a rate of $0.12 \%$ per year ( $p$ trend $=$ 0.001 ). However, despite this increase, the proportion of Black fellows was significantly lower than that of applicants for every year from 2008 to 2016 ( $p<0.035$ for each year) and was only similar in 2017 and 2018 (Fig. 2). The overall proportion of Black resident applicants decreased significantly from 2014 to 2018, at a rate of $0.3 \%$ per year $(p$ trend $=0.01$ ). Our findings indicate that, similar to the female medical population, Black representation decreased when moving from IM residency to fellowship in the most competitive fields $(p<$ 0.001) across the same time period (Fig. 3).

The overall Hispanic proportion of resident applicants and fellows decreased from 2014 to 2018 at a rate of $0.13 \%$ ( $p$ trend $=0.11$ ) and $0.24 \%(p$ trend $=0.04)$ per year respectively. Despite this decrease, the proportion of Hispanic fellows was significantly higher than that of applicants for every year from 2010 to 2018 ( $p<0.01$ for each year) (Fig. 2). Our findings also indicate that Hispanic representation decreased when moving from IM residency to fellowship in the most competitive fields $(p<0.001)$ (Fig. 3). However, Hispanics made up a greater proportion of fellows in the most competitive fields (6.2-7.8\%) as compared with applicants to those fields across the same time period (5.2-6.5\%, $p<0.001)$ (Fig. 2).

\section{Analysis by Specialty}

Gastroenterology. The female proportion of GI fellows significantly increased from 2008 to 2018 ( $p$ trend $=0.007$ ). However, most of this increase occurred between 2008 and 2013 ( $p$ trend $=0.008$ ). Since then, female representation has significantly decreased from 2014 to 2018 ( $p$ trend $=0.006$ ). The Black proportion of fellows significantly increased from 2008 to 2018 ( $p$ trend $=0.001$ ). There were no significant trends over time or differences between the proportions of Hispanic applicants and fellows from 2008 to 2018. Despite this, females and Hispanics in 2018 were each 
Table 1 Aggregate Minority Representation Among Resident Applicants and Accepted Fellows to the Top Five Most Competitive IM Fellowships

\begin{tabular}{|c|c|c|c|c|}
\hline & & Resident applicants* & Fellows* & $p$ value \\
\hline \multirow[t]{10}{*}{ Female } & 2008 & $31.6 \%(1429 / 4518)$ & $29.9 \%(1961 / 6568)$ & 0.049 \\
\hline & 2009 & $32.6 \%(1455 / 4466)$ & $31.2 \%(2121 / 6808)$ & 0.116 \\
\hline & 2010 & $34.2 \%(1496 / 4374)$ & $32.8 \%(2240 / 6831)$ & 0.124 \\
\hline & 2011 & $33.8 \%(1475 / 4367)$ & $33.4 \%(2344 / 7009)$ & 0.071 \\
\hline & 2013 & $33.6 \%(1457 / 4342)$ & $34.3 \%(2513 / 7316)$ & 0.385 \\
\hline & 2014 & $31.7 \%(1334 / 4213)$ & $34.2 \%(2569 / 7503)$ & 0.005 \\
\hline & 2015 & $31.1 \%(1348 / 4338)$ & $33.7 \%(2557 / 7595)$ & 0.015 \\
\hline & 2016 & $31.1 \%(1340 / 4312)$ & $33.5 \%(2559 / 7649)$ & 0.022 \\
\hline & 2017 & $34.5 \%(1515 / 4397)$ & $32.9 \%(2583 / 7856)$ & 0.019 \\
\hline & 2018 & $34.4 \%(1638 / 4760)$ & $33.5 \%(2723 / 8133)$ & 0.073 \\
\hline \multirow{10}{*}{ Black } & 2008 & $4.8 \%(219 / 4518)$ & $3.4 \%(223 / 6568)$ & $<0.001$ \\
\hline & 2009 & $4.9 \%(217 / 4466)$ & $4.0 \%(270 / 6808)$ & 0.035 \\
\hline & 2010 & $5.1 \%(224 / 4374)$ & $3.8 \%(261 / 6831)$ & $<0.001$ \\
\hline & 2011 & $5.4 \%(236 / 4367)$ & $3.8 \%(268 / 7009)$ & $<0.001$ \\
\hline & 2013 & $5.8 \%(252 / 4342)$ & $4.5 \%(330 / 7316)$ & $<0.001$ \\
\hline & 2014 & $6.4 \%(212 / 4213)$ & $4.7 \%(352 / 7503)$ & $<0.001$ \\
\hline & 2015 & $6.2 \%(216 / 4338)$ & $4.5 \%(339 / 7595)$ & $<0.001$ \\
\hline & 2016 & $5.7 \%(201 / 4312)$ & $4.7 \%(360 / 7649)$ & 0.012 \\
\hline & 2017 & $5.2 \%(186 / 4397)$ & $4.7 \%(367 / 7856)$ & 0.191 \\
\hline & 2018 & $5.3 \%(205 / 4760)$ & $4.6 \%(376 / 8133)$ & 0.115 \\
\hline \multirow[t]{10}{*}{ Hispanic } & 2008 & $5.8 \%(260 / 4518)$ & $6.2 \%(410 / 6568)$ & 0.318 \\
\hline & 2009 & $6.5 \%(290 / 4466)$ & $7.4 \%(505 / 6808)$ & 0.064 \\
\hline & 2010 & $6.3 \%(274 / 4374)$ & $7.7 \%(525 / 6831)$ & 0.005 \\
\hline & 2011 & $5.9 \%(258 / 4367)$ & $7.6 \%(535 / 7009)$ & $<0.001$ \\
\hline & 2013 & $5.9 \%(257 / 4342)$ & $7.7 \%(560 / 7316)$ & 0.006 \\
\hline & 2014 & $5.7 \%(189 / 3330)$ & $7.1 \%(530 / 7503)$ & $<0.001$ \\
\hline & 2015 & $5.9 \%(205 / 3491)$ & $7.2 \%(545 / 7595)$ & $<0.001$ \\
\hline & 2016 & $5.8 \%(204 / 3508)$ & $6.7 \%(511 / 7649)$ & $<0.001$ \\
\hline & 2017 & $5.6 \%(199 / 3559)$ & $6.6 \%(515 / 7856)$ & $<0.001$ \\
\hline & 2018 & $5.2 \%(198 / 3840)$ & $6.2 \%(508 / 8133)$ & $<0.001$ \\
\hline
\end{tabular}

* "Resident applicants" refers to all categorical internal medicine residents applying to the top 5 most competitive internal medicine fellowships. "Fellows" refers to all fellows within the top 5 most competitive internal medicine fellowships

$P<0.05$ represents significantly different representation between resident applicants to and fellows among the top 5 most competitive internal medicine fellowships for each respective race and year

underrepresented as a percentage of GI fellows (F 33.6\%; H $6.6 \%)$ as compared with the percentage of categorical internal medicine residents (F 42.8\%, $p<0.001$; H 7.5\%, $p<0.001$ ).
Rheumatology. There were no significant trends over time or differences between the proportions of female, Black, or Hispanic applicants and fellows from 2008 to 2018. The

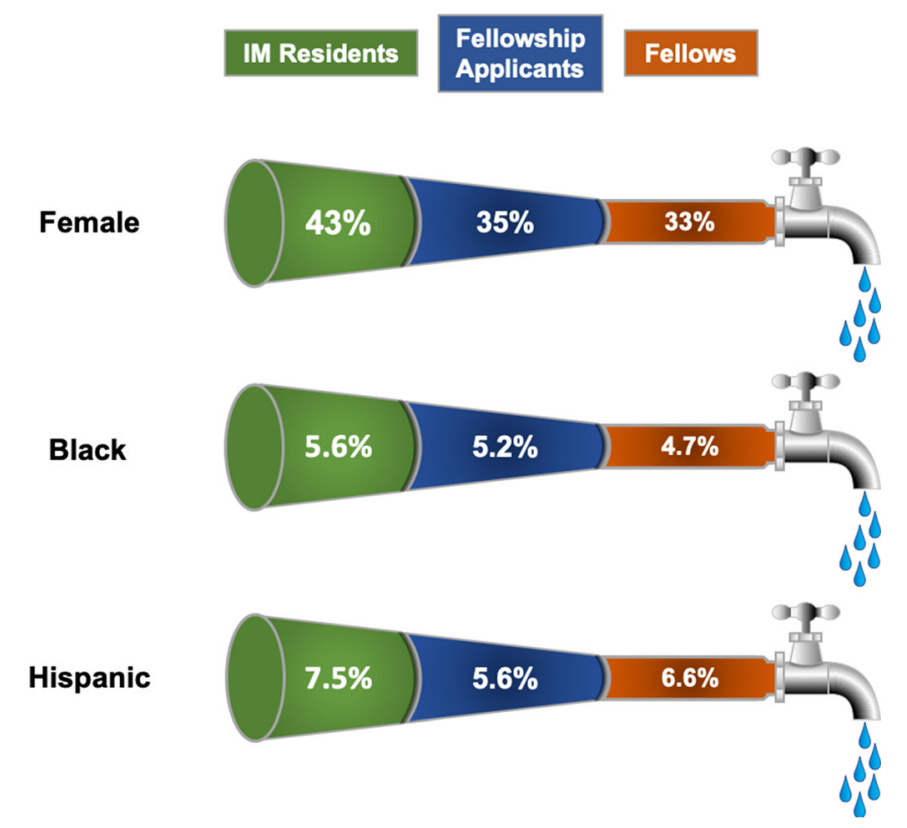

Fig. 1 Female, Black, and Hispanic representation at each stage of training from categorical internal medicine residents to resident applicants to fellows in the top 5 most competitive IM specialties in 2017. All groups continue to face decreasing representation when moving from IM residency to fellowship in the most competitive fields. 

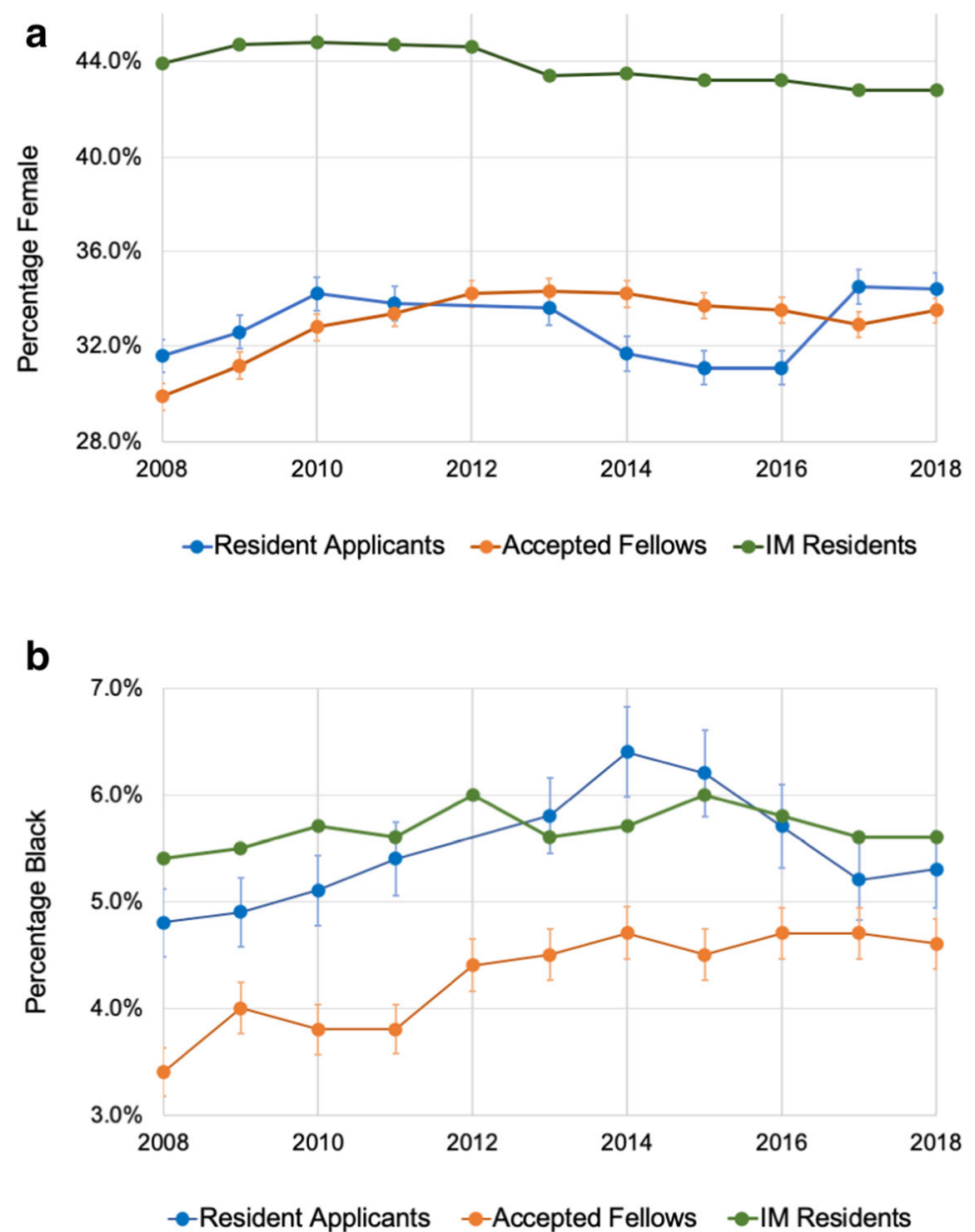

C

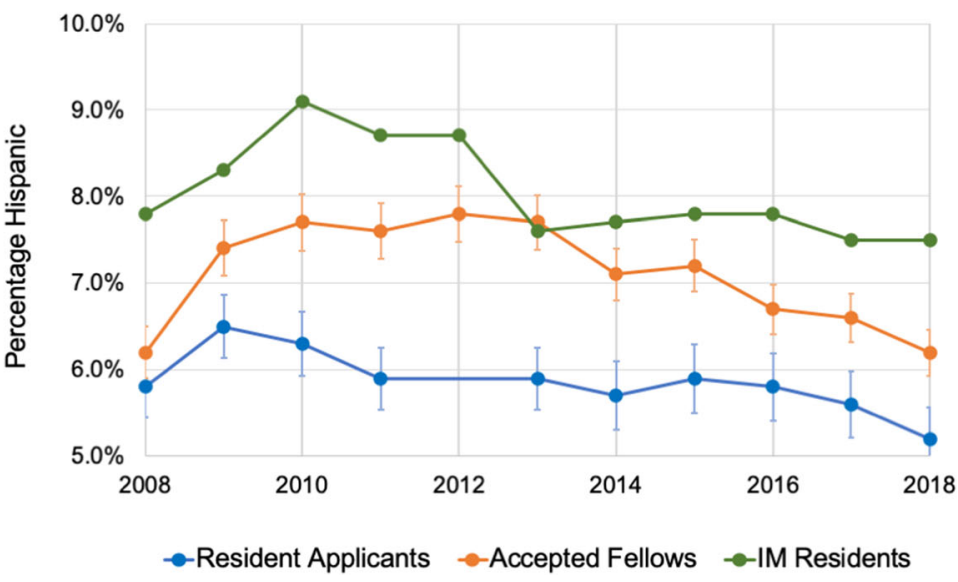

Fig. 2 Female (a), Black (b), and Hispanic (c) representation at each stage of training from internal medicine residents to fellows in the top 5 most competitive IM specialties from 2008 to 2018. The data show a plateau in resident applicants to fellowships and fellows among all three groups from 2014 to 2018 at levels of representation lower than at the categorical IM residency level.

proportion of females among RHEUM fellows $(60.8 \%)$ in 2018 was significantly higher than among categorical internal medicine residents $(42.8 \%, p<0.001)$.

Cardiovascular Disease. The female proportion of CVD fellows significantly increased from 2008 to 2018 ( $p$ trend = 0.03 ). There was no significant difference between the proportion of females among resident applicants and fellows from 2008 to 2016, and 2018. The proportion of Black CVD fellows significantly increased from 2008 to 2018 ( $p$ trend $=$ 0.02 ). Of note, there was no significant difference between the proportion of Black resident CVD applicants and fellows from 2008 to 2018. Female and Hispanic representation in 2018 was significantly lower among CVD fellows (F 22.9\%; H 

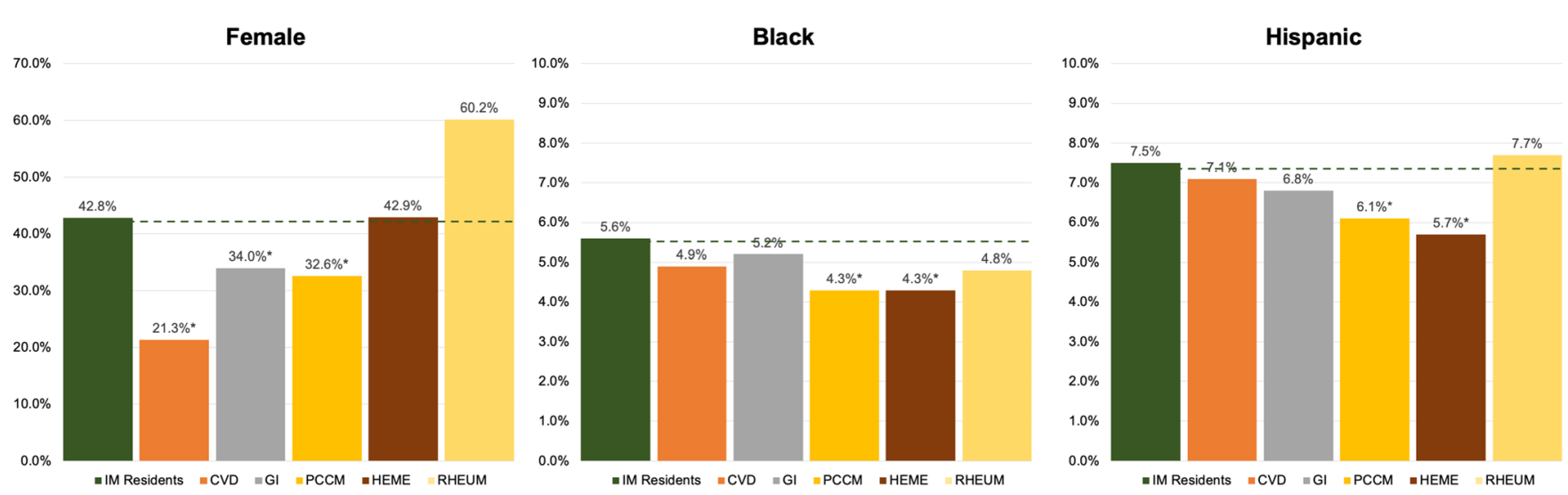

Fig. 3 Female, Black, and Hispanic representation from categorical IM residents to accepted fellows in the top 5 most competitive IM specialties broken down by fellowship for 2017. Dotted line indicates representation at the IM resident level. *Significantly lower representation than at the IM resident level $(p<0.05)$.

6.4\%) than among categorical internal medicine residents $(\mathrm{F}$ $42.8 \%, p<0.001 ; \mathrm{H} 7.5 \%, p<0.001)$.

Pulmonary Disease and Critical Care Medicine. The proportion of female PCCM fellows significantly increased from 2008 to 2018 ( $p$ trend $=0.02$ ). However, most of this increase occurred between 2008 and 2013 ( $p$ trend $=0.005$ ). Since then, female representation has plateaued from 2014 to 2018. The proportion of Black PCCM fellows significantly increased from 2008 to $2018(p<0.001)$. The proportion of Black PCCM applicants significantly decreased from 2014 to 2018 ( $p$ trend $=0.02$ ). The proportion of Hispanic PCCM applicants and fellows significantly decreased from 2008 to 2018 ( $p$ trend $=0.001$ and 0.03 , respectively). Female, Black, and Hispanic representation in 2018 was significantly lower among PCCM fellows (F 33.0\%; AA 4.1\%; H 6.2\%) than among categorical internal medicine residents (F 42.8\%, $p<$ 0.001 ; AA $5.6 \%, p<0.001 ; \mathrm{H} 7.5 \%, p<0.001$ ).

Hematology and Oncology. There were no significant trends over time or differences between the proportion of female applicants and female HEME fellows from 2008 to 2018. The proportion of Black resident applicants significantly increased from 2008 to 2018 ( $p$ trend $=0.008$ ). There were no significant trends over time or differences between the proportions of Hispanic HEME applicants and fellows from 2008 to 2018. Representation of Blacks and Hispanics in 2018 was significantly lower among HEME fellows (AA 3.8\%; H $5.4 \%$ ) than among categorical internal medicine residents (AA $5.6 \%, p<0.001 ; \mathrm{H} 7.5 \%, p<0.001)$.

\section{DISCUSSION}

Increasing racial, ethnic, and gender diversity in training programs is essential for addressing health disparities, improving physician distribution, and providing optimal care to an increasingly diverse patient population. ${ }^{5,26-28}$ Yet, our findings indicate that women and URMs decrease in representation when moving from medical school to IM residency to fellowship programs in the most competitive specialties. Additionally, while the overall trends suggest increasing representation of these groups in fellowship programs, further scrutiny indicates a lack of progress within recent years.

We found that women and URMs were significantly underrepresented as both resident applicants and accepted fellows when compared with IM residents. While we observed an overall increase in the proportion of female fellows between 2008 and 2018, suggesting a shift toward better representation, almost all of this was achieved from 2008 to 2013, with no significant uptrend from 2014 to 2018. This plateau is concerning, as the representation of women in these fellowship programs between these years is still markedly lower than the proportion of female IM residents. We observed similar trends with Black fellows from 2008 to 2018, with significantly lower representation among fellows as compared with IM residents. Of note, the proportion of Black and Hispanic applicants to these fellowships dropped significantly after 2014 despite an increase in fourth-year medical students from these groups applying into categorical IM residency programs. These trends highlight a need to understand factors beyond the medical school level that influence choice of specialty and barriers that prevent women and URMs from applying and being accepted into the most competitive fellowships.

\section{Barriers to Diversity}

One potential barrier to increasing diversity within these specialties is a lack of role models within the field. ${ }^{29}$ Historically, leadership positions within these fields have been predominantly male and white. ${ }^{30,31}$ In fact, only $4 \%$ of full-time faculty physicians identify themselves as either female or URM, and only $3 \%$ of department chairs are women of color. ${ }^{2}$ This unfortunate underrepresentation among leadership in historically male-dominated fields may explain the decreased number of overall applicants. However, in addition to 
advocating for representation of women and URMs in mentor roles, it is also important to ensure mentees are comfortable seeking mentorship from those of other genders and racial backgrounds, to more quickly bridge this gap. Furthermore, existing underrepresentation may further disincentivize future applicants, ${ }^{13}$ making it more difficult to break the cycle of certain fields being "male" or "non-minority" fields. Further research must be conducted on the effects pigeonholing may have in limiting diversity.

Implicit biases may also explain the decreasing representation of women and URMs from medical school through fellowship. An analysis of medical student performance evaluations (MSPE) for residency applications revealed discrepancies in the words used to describe equally performing students of different genders and races/ethnicities. ${ }^{32}$ Black applicants were more likely to be described as "competent," while White colleagues were more often described as "standouts," "exceptional," and "best." This pattern of racial and gender bias in trainee assessment continues in residency, with female residents more often described using nurturing keywords rather than those characterizing clinical acumen and given lower levels than male residents in ACGME Milestone-based assessments. $^{32,33}$ The impact of these biases likely extends into the fellowship admission process, contributing to the observed representation gap between resident applicants and accepted fellows. In addition to being subject to implicit biases, URMs in medicine are disproportionately subject to microaggressions in the workplace, assumptions of lower status, and unfairly tasked as race/ethnicity ambassadors. ${ }^{29}$ Continued exposure to such microaggressions and implicit and explicit biases may motivate females and URMs to pursue historically better represented and concurrently less competitive and lower paying fields.

Adding to the problem, competitive specialties by nature have appeared less amenable to maintaining a work-life balance for both fellows and practicing physicians. On average, physicians in these fields may work upwards of 60 clinical hours a week, not including the time required for administrative and academic responsibilities. ${ }^{13,14,31}$ Therefore, it is understandable that balancing work and personal/family responsibilities can be difficult, especially since these responsibilities regrettably tend to fall disproportionately on women. Compared with male physicians, female physicians are significantly more likely to have partners who were employed fulltime. ${ }^{34}$ It is not surprising, then, that a majority of female physicians report switching to part-time or considering parttime work within 6 years of completing medical training. ${ }^{35}$ As a result, specialties that have a better perceived work-life balance have more equal representation among applicants and accepted fellows. ${ }^{36}$

Competitive specialties have frequently acknowledged the problem of decreased diversity and are starting to implement multiple initiatives to increase representation, including workshops and committees designed to improve bias literacy and equality within academia and clinical practice. ${ }^{12,37-39} \mathrm{An}$ analysis of the efficacy of bias literacy workshops for university faculty demonstrated increased gender-based bias awareness among participants and changes in behaviors following the workshop. ${ }^{40}$ Based on this study, the University of Wisconsin-Madison launched the Bias Reduction in Internal Medicine initiative, a 3-h workshop and modules, to reduce stereotype-based bias and promote workforce diversity using targeted educational interventions. ${ }^{41}$ While these first steps may lead to improved representation, our data show a more recent stagnation in both gender and racial diversity at the fellowship level relative to internal medicine residency and medical school. Although it is unclear why this stagnation occurred from 2014 to 2018, it demonstrates that current efforts have not yet translated into meaningful increases in diversity at the fellowship level.

To be most effective, these changes must start at the highest of levels. By ensuring better representation of women and URMs in academic faculty positions and leadership within professional societies, future physicians will no longer face the dearth of mentors present today. At the individual practice level, policies must be enacted to increase support for workfamily balance, including mindful scheduling of conferences and career development opportunities. At the fellowship application level, removing all gender and racial/ethnic identifiers along with the removal of photos may help mitigate the influence of implicit biases. Further studies examining the effects of these proposed changes and the many ongoing interventions may provide insight into how we can best increase diversity within the most competitive IM specialties.

\section{Study Limitations}

There are several limitations to our study. First, the data obtained are self-reported and may not accurately reflect the actual composition of residents and fellows. However, the AAMC provides the most comprehensive data to date. Second, there is no IM fellowship applicant data available for 2012 (Table 2), as this was the year the task force of the Alliance for Academic IM changed the IM fellowship timeline from an 18-month cycle to a 12-month cycle. Third, prior to 2013, the AAMC provided self-reported demographic data for all applicants regardless of citizenship status, and race and ethnicity were asked as separate questions. This changed in 2014, when the AAMC began to release demographic data for only US citizens and permanent residents and included Hispanic as a race rather than ethnicity. ${ }^{15}$ This change in reporting method may slightly affect proportions of applicants from each race, but primarily affected the proportion of Asian applicants, who were excluded from our analysis.

\section{CONCLUSION}

Despite trends towards better representation among women and URMs among fellows in the most competitive IM specialties from 2008 to 2013, there has been a recent stagnation 
Table 2 Minority Representation Trends from 2008 to 2018 Using Linear Regression Analysis

\begin{tabular}{|c|c|c|c|c|c|c|c|}
\hline \multirow[t]{2}{*}{ Trendline } & & \multicolumn{2}{|l|}{ Female } & \multicolumn{2}{|l|}{ Black } & \multicolumn{2}{|c|}{ Hispanic } \\
\hline & & $+/-$ & $p$ value & $+/-$ & $p$ value & $+1-$ & $p$ value \\
\hline \multirow[t]{3}{*}{ Categorical IM residents } & $2008-2013$ & $-0.5 \%$ & 0.507 & $+0.3 \%$ & 0.269 & $-0.2 \%$ & 0.814 \\
\hline & 2014-2018 & $-0.6 \%$ & 0.029 & $0.0 \%$ & 0.731 & $-0.1 \%$ & 0.520 \\
\hline & 2008-2018 & $-1.1 \%$ & 0.036 & $+0.3 \%$ & 0.037 & $-0.3 \%$ & 0.074 \\
\hline \multirow{3}{*}{ Resident applicants to fellowship } & 2008-2013 & $+0.1 \%$ & 0.191 & $+1.5 \%$ & 0.005 & $-0.1 \%$ & 0.636 \\
\hline & 2014-2018 & $+2.7 \%$ & 0.105 & $-1.0 \%$ & 0.011 & $-0.5 \%$ & 0.109 \\
\hline & $2008-2018$ & $+2.8 \%$ & 0.980 & $+0.5 \%$ & 0.108 & $-0.6 \%$ & 0.069 \\
\hline \multirow[t]{3}{*}{ Fellows } & 2008-2013 & $+4.4 \%$ & 0.011 & $+1.3 \%$ & 0.051 & $+0.8 \%$ & 0.199 \\
\hline & 2014-2018 & $-0.8 \%$ & 0.149 & $-0.1 \%$ & 0.058 & $-0.8 \%$ & 0.039 \\
\hline & 2008-2018 & $+3.6 \%$ & 0.019 & $+1.2 \%$ & 0.001 & $0.0 \%$ & 0.599 \\
\hline
\end{tabular}

The above data show trends in racial and gender representation from 2008 to 2018 at the categorical IM resident level, fellowship applicant level to the five most competitive IM fellowships, and fellow level at the five most competitive IM fellowships. Of note, fellow data includes fellows at all years of training, and not just first-year fellows. Data from 2012 was unavailable

in both gender and racial diversity over the past 5 years. Our findings suggest that women and URMs continue to face significant barriers resulting in decreasing representation when moving from IM residency to fellowship in the most competitive fields. Further research and efforts must be undertaken to address barriers to entry and to advocate for better representation of women and URMs. Finally, we must re-examine these data over time to ensure we continue on the path to equity.

Acknowledgments: Contributors: None other than the listed authors above.

Corresponding Author: Praneet Mylavarapu, MD; Department of Cardiology, University of California, San Diego, CA, USA (e-mail: pmylavarapu@health.ucsd.edu).

Data Availability: The datasets generated during and/or analyzed during the current study are available in the AAMC ERAS repository: https://www.aamc.org/data-reports/interactive-data/eras-statistics. The data are also available from the corresponding author on reasonable request.

\section{REFERENCES}

1. Association of American Medical Colleges. Diversity in the physician workforce: Facts and figures 2016. Available at: http://www.aamcdiversityfactsandfigures2016.org. Accessed Jun 23, 2019.

2. Lautenberger D, Moses A, Castillo-Page LC. An overview of women fulltime medical school faculty of color. AAMC Analysis in Brief. 2016;16(4): 1-2

3. Musa, D., Schulz, R., Harris, R., Silverman, M., Thomas, S. B. (2009). Trust in the health care system and the use of preventive health services by older black and white adults. American Journal of Public Health, 99, 1293-1299.

4. Saha S, Arbelaez JJ, Cooper LA. Patient-physician relationships and racial disparities in the quality of health care. Am J Public Health. 2003;93(10):1713-1719.

5. Marrast LM, Zallman L, Woolhandler S, Bor DH, McCormick D. Minority physicians' role in the care of underserved patients: diversifying the physician workforce may be key in addressing health disparities. JAMA Intern Med. 2014;174(2):289-291.

6. Saha S, Komaromy M, Koepsell TD, Bindman AB. Patient-Physician Racial Concordance and the Perceived Quality and Use of Health Care. Arch Intern Med. 1999;159(9):997-1004. https://doi.org/10.1001/ archinte.159.9.997

7. Diversity in the Physician Workforce. Facts and Figures 2010. Washington, DC: Association of American Medical Colleges; 2010. https:// www.aamc.org/data/. Accessed Dec 13, 2019.

8. National Resident Matching Program, Charting Outcomes in the Match: Results and Data: 2019 Residency Match. Available at: http://www. nrmp.org/main-residency-match-data/ Accessed Jun 15, 2019.
9. National Resident Matching Program, Charting Outcomes in the Match: NRMP Results and Data Specialties Matching Service, 2019 Appointment Year. Available at: http://www.nrmp.org/fellowship-match-data/ Accessed Jun 15, 2019.

10. King TE Jr, Dickinson TA, DuBose TD Jr, Flack JM, Hellmann DB, Pamies RJ, Todd RF 3rd, Torres EA, Wesson DE. The Case for Diversity In Academic Internal Medicine. Am J Med. 2004;116(4):284-9. https://doi. org/10.1016/j.amjmed.2003.12.015. Review.

11. Wesson DE, King TE Jr, Todd RF, Torres EA, Hellmann DB, Flack JM, Dubose TD Jr, Schuster VL. Achieving Diversity In Academic Internal Medicine: Recommendations For Leaders. Am J Med. 2006;119(1):76-81. https://doi.org/10.1016/j.amjmed.2005.10.032.

12. Lane-Fall MB, Miano TA, Aysola J, Augoustides JGT. Diversity in the Emerging Critical Care Workforce: Analysis of Demographic Trends in Critical Care Fellows From 2004 to 2014. Crit Care Med. 2017;45(5):822827. https://doi.org/10.1097/CCM.0000000000002322.

13. Douglas PS, Rzeszut AK, Bairey Merz CN, Duvernoy CS, Lewis SJ, Walsh MN, Gillam L. Career Preferences and Perceptions of Cardiology Among US Internal Medicine Trainees: Factors Influencing Cardiology Career Choice. JAMA Cardiol. 2018;3(8):682-691. https://doi.org/10.1001/ jamacardio.2018.1279.

14. Burke CA, Sastri SV, Jacobsen G, Arlow FL, Karlstadt RG, Raymond P. Gender Disparity in the Practice of Gastroenterology: the First 5 Years of a Career. Am J Gastroenterol.2005;100(2):259-64. https://doi.org/10. 1111/j.1572-0241.2005.41005.x.

15. Association of American Medical Colleges. ERAS Statistics: Historical specialty specific data, by program. Available at: https://www.aamc.org/ services/eras/stats/359278/stats.html. Accessed May 14, 2019.

16. Brotherton SE, Etzel SI. Graduate medical education, 2008- 2009. JAMA 2009;302(12): 1357-72.

17. Brotherton SE, Etzel SI. Graduate medical education, 2009- 2010. JAMA 2010;304:1255-1270.

18. Brotherton SE, Etzel SI. Graduate medical education, 2010- 2011. JAMA 2011;306:1015-1030.

19. Brotherton SE, Etzel SI. Graduate medical education, 2011- 2012. JAMA 2012;308:2264-2279.

20. Brotherton SE, Etzel SI. Graduate medical education, 2012- 2013. JAMA 2013;310:2328-2346.

21. Brotherton SE, Etzel SI. Graduate medical education, 2013- 2014. JAMA 2014:312:2427-2445.

22. Brotherton SE, Etzel SI. Graduate medical education, 2014- 2015. JAMA 2015;314:2436-2454.

23. Brotherton SE, Etzel SI. Graduate medical education, 2015- 2016. JAMA 2016;316:2291-2310.

24. Brotherton SE, Etzel SI. Graduate medical education, 2016- 2017. JAMA 2017;318: 2368-2387.

25. Brotherton SE, Etzel SI. Graduate medical education, 2017- 2018. JAMA 2018;320(10):1051-1070.

26. U.S. Department of Health and Human Services, Health Resources and Services Administration, Bureau of Health Professions. The rationale for diversity in the health professions: a review of the evidence. Available at: http://www. pipelineeffect.com/wp-content/uploads/2015/04/diversityreviewevidence.pdf. Accessed Dec 1, 2017.

27. Cooper LA, Roter DL, Johnson RL, Ford DE, Steinwachs DM, Powe NR Patient-centered communication, ratings of care, and concordance of patient and physician race. Ann Intern Med. 2003;139:907-915. 
28. Xierali IM, Castillo-Page L, Conrad S, Nivet MA. Analysis in Brief: analyzing physician workforce racial and ethnic composition associations: geographic distribution (part II). Association of American Medical Colleges. 2014

29. Osseo-Asare A, Balasuriya L, Huot SJ, et al. Minority resident physicians' views on the role of race/ethnicity in their training experiences in the workplace. JAMA Netw Open. Published online September 28, 2018;1(5):e182723.

30. Wehner MR, Nead KT, Linos K, Linos E. Plenty of moustaches but not enough women: cross sectional study of medical leaders. BMJ. 2015;351:h6311.

31. Shukla R. Current challenges facing women in gastroenterology: how do we move forward?. ACG Case Rep J. 2016;3(3):144-5.

32. Ross DA, Boatright D, Nunez-smith M, Jordan A, Chekroud A, Moore EZ. Differences in words used to describe racial and gender groups in Medical Student Performance Evaluations. PLoS ONE. 2017;12(8):e0181659.

33. Klein R, Julian KA, Snyder ED, et al. Gender bias in resident assessment in graduate medical education: review of the literature. J Gen Intern Med. 2019;34(5):712-719

34. Jolly S, Griffith KA, DeCatro R, Stewart A, Ubel P, Jagsi R. Gender differences in time spent on parenting and domestic responsibilities by high-achieving young physician-researchers. Ann Intern Med. 2014; 160(5):344-356.

35. Frank E, Zhao Z, Sen S, Guille C. Gender disparities in work and parental status among early career physicians. 2019;2(8):e198340

36. American Medical Association. How Medical Specialties vary by Gender Available at: https://www.ama-assn.org/residents-students/specialtyprofiles/how-medical-specialties-vary-gender. Accessed Jun 23, 2019.
37. American College of Cardiology. Inequity in Cardiovascular Medicine: What Can We Do in Our Practice? Available at: https://www.acc.org/ latest-in-cardiology/articles/2017/05/31/17/42/inequity-in-cardiovascular-medicine-what-can-we-do-in-our-practice. Accessed Jun 23, 2019

38. Carethers JM, Quezada SM, Carr RM, Day LW. Diversity within US gastroenterology physician practices: the pipeline, cultural competencies, and gastroenterology societies approaches. Gastroenterology. 2019;156(4):829-833.

39. Butkus R, Serchen J, Moyer DV, Bornstein SS, Hingle ST. Achieving gender equity in physician compensation and career advancement: a position paper of the American College of Physicians. Ann Intern Med. 2018;168(10):721-723.

40. Carnes $\mathrm{M}$, Devine $\mathrm{PG}$, Isaac $\mathrm{C}$, et al. Promoting institutional change through bias literacy. Journal of diversity in higher education. 2012;5(2):63-77.

41. Carnes M. Bias Reduction in Internal Medicine [Internet]. St. Louis (MO): Washington University School of Medicine, Office of Inclusion and Diversity; 2019 [Updated 2019; cited 2019 Dec 9]. Available from https://internalmedicinediversity.wustl.edu/training/bias-reduction-ininternal-medicine-brim/. Accessed 9 Sep 2019.

Publisher's Note: Springer Nature remains neutral with regard to jurisdictional claims in published maps and institutional affiliations. 